\title{
COLLECTIONS FILLING UP A SIMPLE PLANE WEB
}

\author{
R. H. BING
}

A web has been defined [2] $]^{1}$ by R. L. Moore. A compact plane continuum $W$ is said [1] to be a simple plane web if there exist an upper semicontinuous collection $G$ of mutually exclusive continua filling up $W$ and another such collection $H$ also filling up $W$ such that (1) $G$ is a dendron with respect to its elements and so is $H$ and (2) if $g$ and $h$ are elements of $G$ and $H$ respectively, the common part of $g$ and $h$ exists and is totally disconnected. It has been shown [1] that we have an equivalent definition if we substitute for (1) in the above definition the condition that $\left(1^{\prime}\right) G$ is a dendron with respect to its elements and $H$ is an arc with respect to its elements. The present paper shows that an equivalent definition is obtained if condition (1) is omitted.

This paper gives conditions under which the collections filling up a simple plane web are non-equicontinuous. A collection of mutually exclusive continua is said to be equicontinuous if for every positive number $\epsilon$, there is a positive number $\delta_{\epsilon}$ such that if $P$ and $Q$ are points of an element $g$ of $G$ at a distance apart of less than $\delta_{\epsilon}$, there is an arc from $P$ to $Q$ in $g$ of diameter less than $\epsilon$. If a collection is not equicontinuous, it is said to be non-equicontinuous. If there exists a point $P$ belonging to the limiting set of $G$ such that every subcollection of $G$ whose limiting set contains $P$ is non-equicontinuous, then $G$ is said to be hereditarily non-equicontinuous at $P$.

I wish to thank Professor R. L. Moore for his valuable suggestions in the preparation of this paper.

A simple plane web is a continuous curve [3]. In establishing some of the following theorems, we shall make use of the fact [1, Theorem 1] that a necessary and sufficient condition that a continuous curve be a simple plane web is that it remain connected and locally connected on the omission of any countable set of points.

THEOREM 1. A necessary and sufficient condition that a compact plane continuum $W$ be a simple plane web is that there exist an upper semicontinuous collection $G$ of mutually exclusive continua filling up $W$ and another such collection $H$ filling $u p W$ such that if $g$ and h are elements of $G$ and $H$ respectively, the common part of $g$ and $h$ exists and is totally disconnected. 1945.

Presented to the Society, September 17, 1945; received by the editors June 25,

${ }^{1}$ Numbers in brackets refer to the references cited at the end of the paper. 
Proof. The necessity of the above condition follows from the definition of a simple plane web. To demonstrate its sufficiency, we shall show that if $Z$ is a countable subset of $W, W-Z$ is connected and locally connected. The argument is similar to that used in proving the necessity of Theorem 1 of [1].

Let $M$ be the sum of all elements of $G$ that do not intersect $Z$ and let $N$ be the sum of all elements of $H$ that do not intersect $Z$. As $M+N$ is connected and dense in $W, W-Z$ is connected.

Assume that $W-Z$ is not locally connected. Let $d$ be a domain containing a point $P$ of $W-Z$ such that any domain $d^{\prime}$ containing $P$ contains points of an infinitude of components of $(W-Z) \cdot d$. An infinitude of such components contain points of $M \cdot d^{\prime}$. Let $C_{1}, C_{2}, C_{3}$ be circles in $d$ such that $C_{1}$ encloses $P, C_{2}$ encloses $C_{1}$, and $C_{3}$ encloses $C_{2}$ but no element of $G$ or $H$. Then $C_{1}$ encloses a sequence of points $P_{1}, P_{2}, \cdots$ of $M$ such that (1) no two of them belong to the same component of $(W-Z) \cdot d$ and (2) if $g_{n}$ is the component containing $P_{n}$ of the common part of $d$ and the element of $G$ containing $P_{n}$, then $g_{1}, g_{2}, \ldots$ has a sequential limiting set. There exists a sequence of points $Q_{1}, Q_{2}, \cdots$ of $N$ converging to a point of $C_{2}$ such that $Q_{n}$ is a point of a continuum in $g_{m}(m \geqq n)$ irreducible from $C_{1}$ to $C_{3}$. Let $h_{n}$ be the component containing $Q_{n}$ of the common part of $d$ and the element of $H$ containing $Q_{n}$. It follows [2, pp. 391-392] that some nondegenerate continuum $F$ is a subset of the common part of the limiting set of $g_{1}, g_{2}, \ldots$ and the limiting set of $h_{1}, h_{2}, \ldots$ As $G$ and $H$ are upper semicontinuous collections, $F$ is a subset of the common part of an element of $G$ and an element of $H$. This is contrary to the hypothesis of this theorem.

THEOREM 2. If $x$ is the closure of a connected set which is the sum of a countable number of points and complementary domains of a simple plane web $W$, the common part of $W$ and $x$ is a continuous curve. ${ }^{2}$

Proof. It has been shown [1, Theorem 6] that $x$ is locally connected. As $W$ is a continuous curve, for any positive number $\epsilon$ there are only a finite number of complementary domains of $W$ of diameter more than $\epsilon$. Since $W \cdot x$ is equal to $x$ minus the sum of a set of complementary domains of $W$, then $W \cdot x$ is a continuous curve.

${ }^{2}$ Indeed, $W \cdot x$ may be shown to be hereditarily a continuous curve. Furthermore, it may be shown that in order that the compact point set $K$ should be the boundary of the closure of a connected point set which is the sum of a countable number of points and complementary domains of some simple plane web $W$ it is necessary and sufficient that $K$ should be a continuous curve such that (1) every nondegenerate cyclic element of $K$ is a simple closed curve, (2) $K$ does not have uncountably many cut points, and (3) no cyclic element of $K$ encloses a point of $K$. 
THEOREM 3. If $x$ is the closure of a connected set which is the sum of a countable number of points and complementary domains of a simple plane web $W$ and $R$ is a nondegenerate connected subset of the common part of $W$ and $x$, then $R$ is arcwise connected. ${ }^{3}$

Proof. Let $A$ and $B$ be two points of $R$ and let $A B$ be an arc in $W \cdot x$. If $A B$ contains a point $P$ not of $R$, there is an arc $A O B$ in $W \cdot x-P$. There is a simple closed curve which is the sum of arcs $A^{\prime} P B^{\prime}$ and $A^{\prime} Q B^{\prime}$ of $A B$ and $A O B$ respectively. It has been shown $[1$, Theorem 8$]$ that each point of $W \cdot x$ is a limit point of the connected set $W-W \cdot x$. Therefore, no subset of $W \cdot x$ contains a simple closed curve plus an arc having only its end points on the simple closed curve. Using this fact and the fact that there is an arc from $A$ to $B$ in $W \cdot x$ minus a finite number of points not belonging to $R$, we find that $A^{\prime} Q B^{\prime}$ is a subset of $R$. By replacing $A^{\prime} P B^{\prime}$ and other subarcs of $A B$ by arcs which are subsets of $R$, we may obtain an arc from $A$ to $B$ in $R$.

THEOREM 4. If $x$ is the closure of a connected set which is the sum of a countable number of points and complementary domains of a simple plane web $W$ and $A B$ is an arc lying in the common part of $W$ and $x$, then $A B$ contains an arc lying on the boundary of some complementary domain of $W$.

Proof. Since $A B$ is $[1$, Theorem 7$]$ the sum of a countable number of points and closures of individual complementary domains of $W$, the boundary of some one of these domains contains a subarc of $A B$.

THEOREM 5. Let $G$ be an upper semicontinuous collection of mutually exclusive continua filling up a simple plane web $W$ and let $H$ be another such collection filling up $W$ such that (1) $H$ is a dendron with respect to its elements and (2) if $g$ and $h$ are elements of $G$ and $H$ respectively, the common part of $g$ and $h$ exists and is totally disconnected. Let $g_{1}$ and $g_{2}$ be two elements of $G$ belonging to $x_{1}$ and $x_{2}$ respectively where $x_{i}$ $(i=1,2)$ is the closure of a connected set which is the sum of a countable number of points and complementary domains of $W$. Then $g_{1}+g_{2}$ is a subset of the boundary of a complementary domain of $W$.

Proof. By Theorems 3 and 4 there is a complementary domain $d$ of $W$ such that $g_{1}$ and the boundary of $d$ have an arc in common. The boundary of $d$ intersects two elements $h_{1}$ and $h_{2}$ of $H$ and as $g_{2}$ intersects each of them, each element of $H$ that separates $h_{1}$ from $h_{2}$ in $W$ also disconnects both $g_{2}$ and $W \cdot x_{2}$.

I In fact, $R$ is locally arcwise connected. 
Let $Z$ be a countable set of points such that $Z$ plus the sum of a set of complementary domains of $W$ is a connected set whose closure is $x_{2}$. Then each element of $H$ that separates $h_{1}$ from $h_{2}$ intersects $Z$ or contains a point of $x_{2}$ belonging to the boundary of $d$. As $Z$ is countable and as there are uncountably many elements of $H$ each of which separates $h_{1}$ from $h_{2}$ in $W$, the boundary of $d$ contains uncountably many points of $g_{2}$.

Assume that $g_{i}$ ( $i=1$ or 2 ) is not a subset of the boundary of $d$. By Theorems 3 and 4 there is a complementary domain $d^{\prime}$ of $W$ other than $d$ such that some arc is a subset of the common part of $g_{i}$ and the boundary of $d^{\prime}$. Both $g_{1}$ and $g_{2}$ intersect the boundary of $d^{\prime}$ and by Theorem 3 there are two mutually exclusive arcs in $W \cdot x_{1}$ and $W \cdot x_{2}$ respectively such that each has an end point on the boundary of $d$ and an end point on the boundary of $d^{\prime}$. This is impossible, for no subset of $W \cdot x_{1}+W \cdot x_{2}$ plus the boundaries of $d$ and $d^{\prime}$ is a simple closed curve plus an arc having only its end points on the simple closed curve.

Theorem 6. Suppose $W$ is a simple plane web with two complementary domains. Suppose that $G$ is an upper semicontinuous collection of mutually exclusive continua filling up $W$, that $H$ is another such collection also filling up $W$, that $G$ is a dendron with respect to its elements and so is $H$ and finally that if $g$ and $h$ are elements of $G$ and $H$ respectively, then the common part of $g$ and $h$ exists and is totally disconnected. Then there is an element of $G$ which does not separate $W$ and which is not a subset of the closure of any connected set which is the sum of a countable number of points and complementary domains of $W$.

Proof. Those elements of $G$ that do not separate $W$ are called end elements of $G$. Assume that each end element of $G$ is a subset of the closure of a connected set which is the sum of a countable number of points and complementary domains of $W$. Then by Theorem 5 , if $g_{1}$ and $g_{2}$ are end elements of $G$, there is a complementary domain $d$ of $W$ whose boundary contains $g_{1}$ and $g_{2}$.

Suppose that $g_{3}$ is an end element of $G$ other than $g_{1}$ or $g_{2}$. As the boundaries of two complementary domains of a simple plane web have no more than a point in common, it follows by Theorem 5 that $g_{3}$ is a subset of the boundary of $d$. However, if it were a subset of the boundary of $d$, no two elements of $H$ could intersect each of the sets $g_{1}$, $g_{2}$, and $g_{3}$.

Suppose that $g_{1}$ and $g_{2}$ are the only end elements of $G$. If $d^{\prime}$ is a complementary domain of $W$ other than $d$, its boundary is a subset of an element of $H$, for no three mutually exclusive continua in $W$ could 
intersect the boundary of $d^{\prime}$ as well as both $g_{1}$ and $g_{2}$. Likewise, the boundary of $d^{\prime}$ is a subset of an element of $G$, for no three mutually exclusive continua in $W$ could intersect both the boundary of $d$ and the boundary of $d^{\prime}$. This is impossible as no element of $G$ intersects an element of $H$ in a nondegenerate continuum.

THEOREM 7. Let $W$ be a simple plane web with two complementary domains. Let $G$ be an upper semicontinuous collection of mutually exclusive continua filling $u p W$ and let $H$ be another such collection filling up $W$ such that (1) $G$ is a dendron with respect to its elements and so is $H$ and (2) if $g$ and $h$ are elements of $G$ and $H$ respectively, the common part of $g$ and $h$ exists and is totally disconnected. Then the collection $G$ is non-equicontinuous.

Proof. Let $X$ be a set such that $x$ is an element of $X$ only if $x$ is maximal with respect to being the closure of a connected set which is the sum of a countable number of points and complementary domains of $W$. By Theorem 6 there is an end element $g_{e}$ of $G$ which is not a subset of an element of $X$. There is [1, Theorem 8] an arc $A B$ in $W$ which contains no point of an element of $X$ and which has only its end points in common with $g_{\theta}$.

Let $d$ be one of the complementary domains of $g_{e}+A B$ which has $A B$ as a subset of its boundary. If $F$ is a point of $W$ in $d$ and $E$ is a point of $W$ belonging to neither $d, A B$, nor $g_{e}$, then there is an arc $E F$ in $W$ which contains no point of $A B$. Let $C$ be the first point of the closure of $d$ on $E F$ in the order from $E$ to $F$. If $\rho$ is one-half the distance from $C$ to $A B$, we shall show that for any positive number $\epsilon$ there exist points $P$ and $Q$ belonging to an element $g$ of $G$ such that the distance from $P$ to $Q$ is less than $\epsilon$ and the diameter of any subcontinuum of $g$ containing $P$ and $Q$ is of diameter more than $\rho$.

There exist points $P_{1}$ and $P_{2}$ such that each can be joined to $C$ by an arc in $W$ of diameter less than the minimum of $\rho$ and $\epsilon / 2$ and such that $P_{1}$ belongs to $d$ and $P_{2}$ belongs to neither $d$ nor $g_{e}$. As $g_{e}$ is an end element of $G$, there is an element $g$ of $G$ which separates $g e$ from $P_{1}+P_{2}$ in $W$. There exist on $g$ points $P$ and $Q$ at a distance from $C$ of less than the minimum of $\rho$ and $\epsilon / 2$ such that $P$ belongs to $d$ and $Q$ does not. Every subcontinuum of $g$ that contains $P$ and $Q$ intersects $A B$ and therefore is of diameter more than $\rho$.

THEOREM 8. Let $W$ be a simple plane web with two complementary domains. Let $G$ be an upper semicontinuous collection of mutually exclusive continua filling up $W$ and let $H$ be another such collection filling up $W$ such that (1) $G$ is an arc with respect to its elements, (2) $H$ is a 
dendron with respect to its elements, and (3) if $g$ and h are elements of $G$ and $H$ respectively, the common part of $g$ and $h$ exists and is totally disconnected. Then $W$ contains a point at which $G$ is hereditarily nonequicontinuous.

Proof. Obtain $g_{e}, A B, C, \rho$, and $g$ as in Theorem 7. Of every countable sequence of different elements of $G$ having a subset of $g$ as a limiting set, all but a finite number separate $g$ from $g_{e}$. Hence $G$ is hereditarily non-equicontinuous at $C$.

\section{REFERENCES} Soc.

1. Bing, R. H., Concerning simple plane webs, submitted to Trans. Amer. Math.

2. Moore, R. L., Concerning continua which have dendratomic subsets, Proc. Nat. Acad. Sci. U. S. A. vol. 29 (1943) pp. 384-389.

3. - Concerning webs in the plane, Proc. Nat. Acad. Sci. U. S. A. vol. 29 (1943) pp. 389-393.

The University of Texas

\section{DIMENSIONAL TYPES}

A. D. WALlaCe

Let $H$ and $S$ be topological spaces. We say that $H$ is of dimensional type $S$ (symbol: $D_{S}$ ) if for each closed set $X$ and mapping $f: X \rightarrow S$ there exists an extension $\bar{f}: H \rightarrow S$.

It is clear that (from a result due to Hurewicz [1, p. 83]) when $H$ is separable metric and $S$ is an $n$-sphere, then $H$ can be of dimensional type $S$ if and only if $\operatorname{dim} H \leqq n$. For simplicity we write $D_{n}$ for $D_{S}$ when $S$ is an $n$-sphere. It is, of course, possible to define $\operatorname{dim} H$ as the least integer $n$ for which $H$ is of type $D_{n}$ even when $H$ is not separable metric. But this seems to be open to objection except in certain cases (cf. (d) below).

It is at once clear that we have:

(a) If $H$ is of type $D_{S}$ then so also is any closed subset.

(b) If the closed sets $H_{1}$ and $H_{2}$ are of type $D_{S}$ then so also is the set $H_{1}+H_{2}$.

As a matter of notation we may suppose that $H=H_{1}+H_{2}$. Let $f: X \rightarrow S$. Several cases may arise of which we shall consider only the 1945.

Presented to the Society, September 17, 1945; received by the editors June 13, 